\title{
Editorial: Introducing Roadmaps as special content
}

At PRX Quantum, along with showcasing outstanding research in quantum information science and technology, we are working to create a home for our multidisciplinary research community to collaborate and engage. This community has embraced the challenge of effectively communicating the latest quantum research for the benefit of industry and society. It is part of our mission to serve the scientific community in this way.

Scientists participating in the US National Quantum Initiative came together to discuss plans for the next decade of research, working to establish a unified strategic vision for quantum information science. This group encompassed researchers from across universities, national labs, and industry, and represents a diverse cross section of the US quantum community. Their goal was to converge efforts and accelerate the output of key innovations that will lead to practical quantum technologies. This group has developed a detailed Roadmap, outlining potential ways to work together to tackle the many obstacles ahead. This road will be challenging, and will require the collaborative action of physicists, mathematicians, computer scientists, electrical engineers, materials scientists, and others.

Fulfilling our mission, we are pleased to present the community with the outcomes of this process, published here as a set of Roadmap articles on three key subjects: Quantum Computation [1], Quantum Simulation [2], and Quantum Interconnects [3]. They each represent a call for action. We hope that by enhancing these Roadmaps through the visibility and recognition that come with publication in PRX Quantum, the broader research community will be inspired to join in this exciting interdisciplinary venture.

[1] Y. Alexeev, D. Bacon, K. R. Brown, R. Calderbank, L. D. Carr, F. T. Chong, B. DeMarco, D. Englund, E. Farhi, B. Fefferman et al., Quantum Computer Systems for Scientific Discovery, PRX Quantum 2, 017001 (2021).

[2] E. Altman, K. R. Brown, G. Carleo, L. D. Carr, E. Demler, C. Chin, B. DeMarco, S. E. Economou, M. A. Eriksson, K.-M. C. Fu et al., Quantum Simulators: Architectures and Opportunities, PRX Quantum 2, 017003 (2021).

[3] D. Awschalom, K. K. Berggren, H. Bernien, S. Bhave, L. D. Carr, P. Davids, S. E. Economou, D. Englund, A. Faraon, M. Fejer et al., Development of Quantum Interconnects (QuICs) for Next-Generation, PRX Quantum 2, 017002 (2021).

Stephen Bartlett

Lead Editor

Katiuscia N. Cassemiro

co-Managing Editor

Stojan Rebic

co-Managing Editor

Published 24 February 2021

DOI: 10.1103/PRXQuantum.2.010001

Published by the American Physical Society under the terms of the Creative Commons Attribution 4.0 International license. Further distribution of this work must maintain attribution to the author(s) and the published article's title, journal citation, and DOI. 